\title{
Optically and spatially templated polymer architectures formed by photopolymerization of reactive mesogens in periodically deformed liquid crystals
}

\author{
Vijay Kumar Baliyan, Seung Hee Lee and Shin-Woong Kang
}

A unique and versatile method for forming optically (that is, orientationally) and spatially patterned polymer architectures was developed based on the photopolymerization of reactive mesogens (RMs) in a periodically deformed liquid crystal (LC). Without using lithographic or holographic implements, various polymer patterns were produced by employing nematic LCs as reaction solvents and spatially nonuniform electric fields. The nematic mixture, containing $\mathbf{5 . 0}$ wt.\% RMs and sandwiched between patterned electrodes, was exposed to spatially uniform reaction-initiating radiation. The spatially nonuniform electric field induced periodic optical patterns in the reaction template with spatially varying elastic deformations. The resulting polymerized RM networks were both spatially and optically patterned, with good fidelity with respect to the electrode pattern and subsequent periodic director profiles. The spatial distribution of dense RM networks coincided precisely with the profile of highly deformed regions in the reaction medium. The optical birefringence of the polymer network was templated by the local director of the reaction template. Numerical calculations of director configuration and the associated elastic energy of the reaction template precisely matched the spatial and orientational order of polymerized RM networks. The proposed method provides ease and flexibility in forming organized polymer architectures for functional materials that require both positional and orientational order for their applications.

NPG Asia Materials (2017) 9, e429; doi:10.1038/am.2017.151; published online 25 August 2017

\section{INTRODUCTION}

Processing soft matter with various functionalities is critical for organic electronic and photonic applications. Soft materials provide conformational, processing and design advantages in effectively exploiting the desired properties of small molecules on longer length scales. ${ }^{1,2}$ However, making functional materials is only the first step. To realize and optimize the performance of practical devices, patterning in one or more dimensions is frequently required to achieve both spatial distribution and orientational order in their collective assembly. ${ }^{3-8}$ The materials' functionality and potential for application are severely compromised if they cannot be effectively processed using straightforward techniques.

In this context, liquid crystals (LCs) have attracted great attention as a typical example of self-assembling soft materials with electronic and photonic functionalities. ${ }^{7-10}$ Because of their mesomorphic behaviors, particularly their electrical and optical anisotropies with respect to dynamic fluidity, LCs have been intensively studied for several decades. LC-polymer composites in particular have been broadly investigated for electro-optical applications. ${ }^{11-13}$ Polymerization of reactive mesogens (RMs) in LC phases has been widely explored for the stabilization of particular optical states, for the modification of electro-optical properties and for templating the orientational order of functional materials. ${ }^{11-18}$ Much is known about templating the orientational order of LCs into polymer networks based on the photopolymerization of RMs in an orientationally ordered LC medium. The templated polymer networks exhibit both morphological and orientational anisotropy, mimicking those of the host LCs. ${ }^{11-20}$

However, in a majority of the systems investigated to date, the polymer networks are positionally disordered or ordering is imposed externally by manipulating the intensity distribution of reaction-initiating radiation. To manipulate the spatial distribution of polymer networks, lithographic and holographic techniques have been developed and refined by many researchers. ${ }^{21-24}$ The control of network distribution, perpendicular to the wavefront of reaction-initiating ultraviolet (UV) light, has been demonstrated by manipulating the intensity gradient along the light-propagation direction by using UV-absorbing dye additives or by selecting the appropriate UV wavelength. ${ }^{25-29}$

The formation of both spatially and orientationally structured networks, without using external lithographic or holographic implements, has been demonstrated by using pattern-forming states of cholesteric liquid crystals as reaction templates. ${ }^{30-33}$ It has been hypothesized that the spatial templating is driven by the spatially nonuniform elastic deformation of cholesteric pattern-forming states as a reaction medium. ${ }^{30-32}$ However, this method is rather restrained 

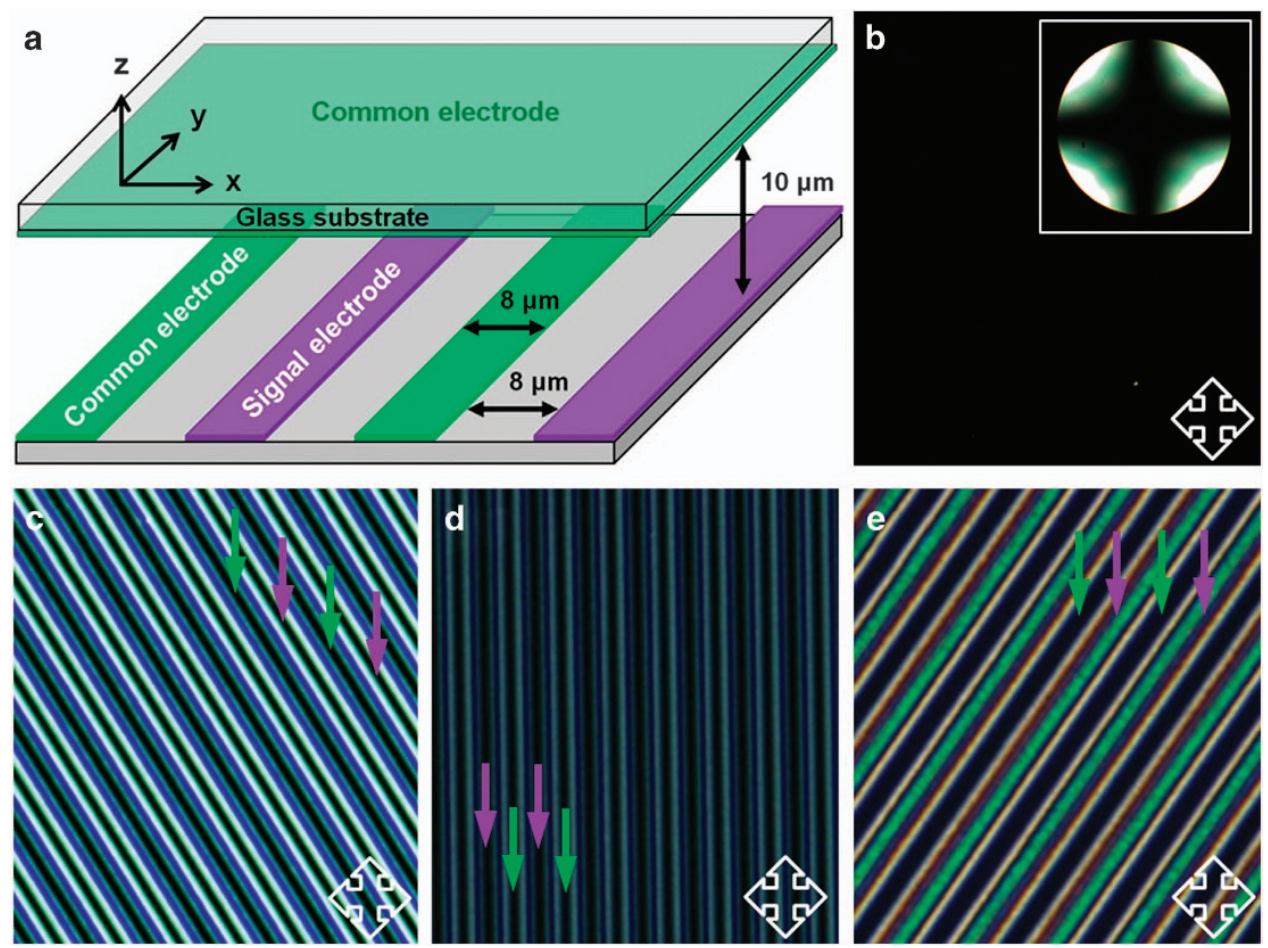

Figure 1 Schematic illustration and polarized optical microscopy (POM) textures of the liquid crystal (LC) cell used as a reaction vessel and medium. (a) Structure of the LC cell used as a reaction vessel for photopolymerization reactions; (b) polarized optical micrograph of the cell, with a conoscopic figure in the inset under no applied electric field; (c, d) polarized optical textures with different orientations at $4.0 \mathrm{~V}_{\mathrm{pp}}$; and (e) polarized optical image after polymerization and removal of applied electric field. In (a), green and purple represent the common and signal electrodes, respectively. A nonpatterned common electrode was used as the top substrate. The green and purple arrows denote the locations of the corresponding common and signal electrodes in (c-e). Crossed double-ended arrows indicate the direction of the transmission axis of the polarizer and analyzer in the POM images. In (c-e), the periodicity of the optical patterns corresponds to $32.0 \mu \mathrm{m}$ that is double that of the electrode pattern.

by the limited number of pattern-forming states of cholesteric liquid crystals. The patterns can be templated only by the available patternforming states of cholesteric LCs.

In this study, both spatially and orientationally structured polymer architectures (that is, morphologically and optically patterned networks) were formed without using externally imposed lithographic or holographic patterning tools. A reaction mixture containing a low concentration of RMs dissolved in a nematic LC was confined by standard LC cells (that is, reaction vessels) with one-dimensionallypatterned transparent electrodes. The spatially nonuniform deformation of nematic LCs was induced by applying a patterned electric field, and the LCs were used as a reaction template. Polymerization of RMs using spatially uniform UV light resulted in morphologically patterned phase separation of the networks. Periodically architectured RM networks with embedded molecular orientational order were constructed and thoroughly examined by polarized optical microscopy (POM) and scanning electron microscopy (SEM).

To elucidate the origin of the templating effect, director configurations and the associated elastic deformations of the reaction template were computed and compared with those of the patterned RM networks. The spatial distribution of dense RM networks coincided precisely with the profile of highly deformed regions in the reaction medium. Our results unambiguously verify the origin of both spatial and orientational templating of polymer networks in periodically deformed LCs. In addition, the proposed method provides flexibility and versatility in constructing various polymer patterns with molecular orientational order by employing patterned electrodes of various shapes and length scales.

\section{MATERIALS AND METHODS}

\section{Materials}

For the anisotropic reaction solvent, nematic LC MLC 15600-100 (Merck Performance Materials, Pyongtaek, Korea) was used as received. 4-(3-Acryloyloxypropyloxy) benzoic acid 2-methyl-1,4-phenylene ester, known as RM-257, was used as a RM that forms polymer networks through photopolymerization. A small amount of IrgaCure 651 was added to initiate the photopolymerization reaction. The RM and photoinitiator were homogeneously dissolved in the host LC in the following weight ratio: host LC (94.75), RM (5.00) and photoinitiator (0.25). For more details on the materials used for the study, see Supplementary Figure S1 in the Supplementary Information.

\section{Sample preparations}

Standard LC cells were fabricated and used as reaction vessels. To fabricate LC cells, both unpatterned and one-dimensionally-patterned indium tin oxide (ITO) glass slides were employed as the top and bottom substrates, respectively. For the patterned bottom substrate, a one-dimensional striped pattern with a $16 \mu \mathrm{m}$ pitch and $8 \mu \mathrm{m}$ width for the ITO electrode was formed in the plane of the substrate, as shown in Figure 1a. The inner surfaces of both substrates were treated with a homeotropic polyimide solution (AL-60702, JSR Micro Korea, Cheongju, Korea) and baked at $180^{\circ} \mathrm{C}$ for $1 \mathrm{~h}$. A cell gap of $\sim 10.0 \mu \mathrm{m}$ between the patterned and unpatterned substrates was maintained using tape as a spacer. The homogeneous reaction mixture was loaded into the cells at an isotropic temperature. The LC cells were cooled to room temperature and used for the photopolymerization reaction.

\section{Polymerization}

Before initiating polymerization, an external electric field was applied to the common and signal electrodes, as shown in Figure 1a, to induce a periodically deformed director profile in the reaction medium. Various amplitudes of a 
square-form electric field were applied at a frequency of $1 \mathrm{kHz}$. The resulting polarized optical patterns were examined by POM, and two voltages (4.0 and $16.0 \mathrm{~V}_{\mathrm{pp}}$ ) were applied to induce distinct optical textures and subsequent polymerization. Under an applied voltage (that is, in the periodically deformed LC solvent), the polymerization of RMs was initiated by UV light (over a wavelength range of 350-390 nm, with a peak at $370 \mathrm{~nm}$ ). The entire cell was illuminated with uniform UV light from the patterned bottom substrate without using lithographic or holographic tools. Polymerization was induced at an intensity of $200 \mu \mathrm{W} \mathrm{cm} \mathrm{cm}^{-2}$ for $1 \mathrm{~h}$.

\section{Characterizations}

The optical and polarized optical characteristics of the LC cells (that is, reaction vessels) and polymer networks were examined using a polarized optical microscope (Nikon Eclipse LV 100 POL, Tokyo, Japan) equipped with a Nikon DS-Ril CCD camera. Before and after polymerization, the optical patterns and corresponding director profiles of the LC cells were carefully investigated and compared with the calculated results and structured polymer networks. To scrutinize both the spatial and orientational order of the structured polymer networks, the LC host was preferentially removed from the crosslinked networks by dissolving in hexane-dichloromethane co-solvent (80/20 wt. ratio). The optical patterns and orientational order of the polymerized RMs were inspected by POM for both assembled cells and dismantled substrates. The morphological patterns and spatial order of the bare networks were investigated using SEM (JEOL JSM-5900/Hitachi, Tokyo, Japan) after depositing a thin platinum film over each sample.

\section{Calculation of elastic energy}

The director configuration and corresponding elastic energy density of the reaction medium at various applied voltages were computed by using the commercial software LCD Master 2D (Shintech, Kumage-Gun, Japan) and confirmed by TechWiz LCD (Sanai System, Incheon, Korea). ${ }^{34,35}$ The resulting two-dimensional director field and associated elastic energy density were used to elucidate the patterned phase separation of polymerized RM networks. Further details can be found in the Supplementary Information.

\section{RESULTS AND DISCUSSION}

To construct three-dimensionally (3D)-architectured polymer networks with optical birefringence, elastically deformed LCs were used as an anisotropic polymerization medium. Standard LC cells were fabricated by combining two glass substrates with a $10 \mu \mathrm{m}$ gap as shown in Figure 1a. The common and signal electrodes are shown in green and purple, respectively. After injecting the reaction mixture into the cell, the LC molecules were aligned vertical to the substrate. The POM image in Figure 1b shows a complete extinction state of the homeotropically aligned LC cell, observed with no applied voltage. The conoscopic figure in the inset confirms the vertical orientation of the uniaxial optic axis (that is, LC director) of the anisotropic reaction mixture. The LC molecules with positive dielectric anisotropy $(\Delta \varepsilon>0)$ were reoriented parallel to the electric field upon the simultaneous application of both in-plane and vertical fields to the common and signal electrodes. As a result, spatially induced birefringence formed a periodic optical pattern along the $x$-direction (that is, across the patterned electrodes). Figures $1 \mathrm{c}$ and d display the POM textures observed at $4.0 \mathrm{~V}_{\mathrm{pp}}$, that show different orientations with respect to the crossed polarizers, marked by double-ended arrows. The green and purple arrows represent the corresponding locations for the common (green) and signal (purple) electrodes at the bottom substrate.

At a given voltage, the areas corresponding to the common and signal electrodes appeared as thick, dark lines (Figures $1 \mathrm{c}$ and $\mathrm{d}$ ) because the LC director retained its homeotropic orientation above the patterned electrodes. Above the signal electrode, the vertical field held the LC molecules in a homeotropic state, whereas the orientation of the LC molecules above the common electrode was not affected because the molecules experienced no effective electric field. However, both edges of the signal electrodes exhibited strong birefringence and thus appeared as bright stripes. The bright stripes near the edges of the signal electrode gradually moved toward to the center of the interelectrode area with an increase in the applied voltage. The periodicity of the optical pattern was $32.0 \mu \mathrm{m}$ that was double the electrode pitch. The overall darker image shown in Figure 1d, compared with that shown in Figure 1c, reveals that the director reorientation occurred along the transverse to the electrodes. Switching between the states illustrated in Figures $1 \mathrm{~b}$ and $\mathrm{c}$ was completely reversible. A similar observation has been reported for display applications with positive $\Delta \varepsilon$ LCs and inplane electrodes with no electrode on the top substrate. ${ }^{36,37}$ In this case, however, the director configuration and periodicity were different because there was no top common electrode.

After polymerization of the RMs, however, the optical pattern was stabilized against the removal of the applied voltage. Figure 1e shows the POM image obtained after polymerization and removal of the electric field. Upon removal of the electric field, the overall optical texture retained its pattern, although slight relaxation of the local director was observed. In Figure 1e, the wide, dark band on the common electrode (marked by the green arrow) corresponds to vertically aligned LCs as the initial state. However, the signal electrode area (purple arrow) in between white stripes is divided into two regions colored green and brown, indicating the asymmetry of the LC orientation induced by the polymerized RM networks. This phenomenon was further confirmed by the structured RM networks discussed in the following paragraph.

Upon uniform UV light irradiation, RM networks were templated by the periodically deformed LC host that was used as a polymerization medium. Figure $2 \mathrm{a}$ shows the director profile and associated elastic deformation of the LC host at $4.0 \mathrm{~V}_{\mathrm{pp}}$. Figures $2 \mathrm{~b}$ and $\mathrm{c}$ show polarized optical and optical patterns of bare polymer networks after polymerization and complete removal of the LC solvent. Most of the networks were formed and bound to the bottom substrate. Negligible amounts of polymer were observed on the top substrate. The dense polymer bundles were located in the interelectrode area (marked by (1), (2), (3)). The weak birefringence of the dense bundles suggested a nearly vertical orientation of the optical axis of the polymerized RMs. The birefringent polymer networks were connected to the dense bundles, with the optical axis along the transverse direction on the side of the signal electrodes. Figure $2 \mathrm{~b}$ shows no evidence of the existence of birefringent networks on either electrode (green and purple dots). Figure 2d shows SEM images of the patterned networks observed on the bottom substrate. A uniform morphological pattern can be observed throughout the entire active area of the cell. The green and purple dots correspond to the regions of the common and signal electrodes, respectively. The SEM images exhibit a continuous polymer layer with periodic dense polymer walls. These POM and SEM results verify that the optical axis of the thin RM network formed on the bottom electrodes was aligned normal to the layer.

Note the unequal distance between dense polymer walls. The distances between (1) and (2) and between (2) and (3) are different in Figure 2. Although the pitch was fixed by the electrode pattern, the location of the dense walls varied with the applied voltage and dielectric anisotropy $(\Delta \varepsilon)$ of the host LCs. At a low voltage and small $\Delta \varepsilon$, walls were formed near the edges of the signal electrode. The displacement of the walls toward the common electrode was proportional to the increase in the applied voltage and $\Delta \varepsilon$. This effect was closely related to the director configuration and its spatial distribution that also depended on the applied voltage and $\Delta \varepsilon$. The images in Figure $2 \mathrm{~d}$, which were obtained at a rotation angle of $45^{\circ}$ in the 

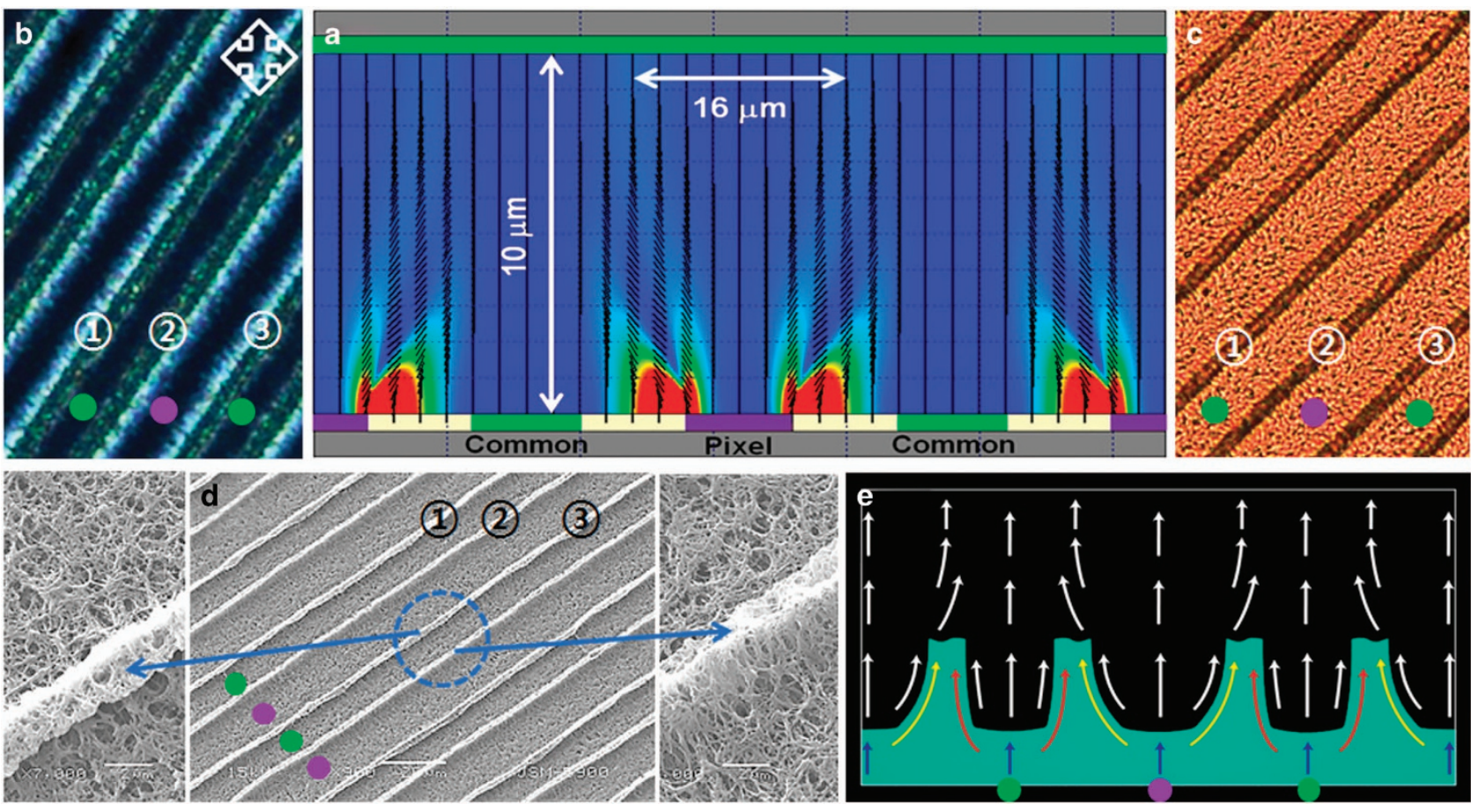

Figure 2 Optically and morphologically templated reactive mesogen (RM) networks formed at $4.0 \mathrm{~V}_{\mathrm{pp}}$. (a) Computed director configuration and associated elastic energy distribution of the polymerization medium, (b) polarized optical and (c) optical images of the polymer networks after polymerization and complete removal of liquid crystal (LCs), (d) scanning electron microscopy (SEM) images of the bare polymer networks formed on the patterned electrodes and (e) schematic illustration of spatial and orientational order of polymer networks along a cross-sectional view. The green and purple dots designate the locations of the common and signal electrodes, respectively. In (a), LC molecules are designated by the thin rods and elastic energy is represented by the color scale. Blue and red correspond to the minimum and maximum elastic energy density, respectively. For the circled area in (d), magnified views of the dense polymer walls are presented as leaning toward the common electrode. The periodicity (11-3) of the polymer patterns corresponds to $32.0 \mu \mathrm{m}$ that is double that of the electrode pattern. In (e), white and colored arrows correspond to the local director profiles for the host LC and polymerized RM networks, respectively.

substrate plane and at a tilt angle of $30^{\circ}$, clearly show the unequal spacing between the walls. This unequal spacing was caused by the tilting of the polymer walls toward the common electrodes. The magnified views of the circled area in Figure $2 \mathrm{~d}$ clearly demonstrate the tilting of the walls away from the signal electrode. This tilted morphology also corroborates the brighter lines observed near the edges of the signal electrodes compared with those near the common electrodes in Figure 2b. The location of the walls was strongly dependent on the applied voltage. The walls gradually shifted toward the common electrode with an increase in voltage.

Figures $2 \mathrm{a}$ and e summarize the results obtained for the patterned phase separation of RM networks formed at $4.0 \mathrm{~V}_{\mathrm{pp}}$ in an anisotropic LC solvent. Figure 2a presents the director configuration (represented by thin solid bars) at $4.0 \mathrm{~V}_{\mathrm{pp}}$ and the corresponding elastic deformation density (scaled by color). Blue and red indicate minimum and maximum elastic energy, respectively. Note the different length scales for the horizontal and vertical directions. At a given voltage, the initial vertical alignment of the LCs was maintained across a wide area above the common electrodes, where no effective field existed (see also Supplementary Figure S3 in the Supplementary Information). Almost no elastic deformation was observed. Meanwhile, the vertical orientation of the director above the signal electrodes was reinforced by the dielectric coupling between the vertical field and the positive dielectric anisotropy $(\Delta \varepsilon>0)$ of the LCs. The reorientation of the LC molecules and the associated elastic deformation mainly occurred in the interelectrode regions because of a lateral in-plane field between the interdigitated electrodes. At a low voltage, a highly deformed region formed near both edges of the signal electrode, as shown in Figure 2a.
The highly deformed region shifted laterally toward the common electrode and expanded toward the top substrate with an increase in the applied voltage (see Supplementary Figure S3 in the Supplementary Information). This finding coincides with the observations discussed above with respect to Figure 1 . The thick white lines near the signal electrode in Figure 1c and the polymer walls in Figure 2c show similar lateral shifts with increasing voltage. The director configuration in Figure 2a well supports the optical patterns shown in Figures $1 \mathrm{c}$ and $\mathrm{d}$. More importantly, the spatial variation of the elastic deformation energy precisely coincides with the observed network morphology. The pattern of the dense polymer walls closely matches the profile of the highly deformed region, shown in red in Figure 2a. The same tilt direction and cleavage at the top of the polymer wall are observed in the highly deformed red region in Figure 2a.

Figure $2 \mathrm{e}$ shows the simplified director orientation of the host LCs (white arrows) and the local orientational order of the RM networks (colored arrows), reconstructed based on POM/SEM observations and the computed director configuration. The more inclined optical axis of the local networks on the side of the signal electrodes (denoted by yellow arrows), compared with that on the common electrode side (red arrows), was responsible for the more birefringent networks shown in Figure 2b. The spatial distribution (that is, morphological pattern) of the RM networks was precisely determined by the elastic deformation of the anisotropic reaction medium. The molecular order (that is, optical birefringence) in local networks was templated by the local director orientation of the LC host used as a reaction medium. The origin of the vertically aligned thin networks (blue arrows) appears to have been different from that of elastic energy-driven 
templating. Because the LC cell was illuminated by polymerizing UV light from the bottom substrate with patterned electrodes, the intensity gradient of UV light inside the cell may have caused preferential polymerization near the front surface of the cell, followed by frontal polymerization. ${ }^{25-29,38,39}$

By increasing the applied voltage, the vertical field in between the signal and top common electrodes strengthened the uniform vertical orientation of LCs with minimum elastic deformation above the signal electrode. At the same time, the increased in-plane field further squeezed the deformed regions toward both the (bottom and top) common electrodes. The director configuration and corresponding elastic energy at $16.0 \mathrm{~V}_{\mathrm{pp}}$ are shown in Figure 3a. The spatial distribution of the elastic deformation is significantly different from that shown in Figure 2a. A highly deformed disclination line (that is, wall in 3D), where the director could not be defined, formed at the center of the bottom common electrode, denoted by the red wavy line in Figure 4a. ${ }^{36,37}$ Disclination lines appeared as thick green lines under POM, as shown in Figure 3b. In Figure 3b, the wide dark bands coincide with the signal electrodes with homeotropic LCs (marked by the purple arrow), and the disclination lines are positioned at the center of the common electrode (marked by the green arrow). ${ }^{36,37}$
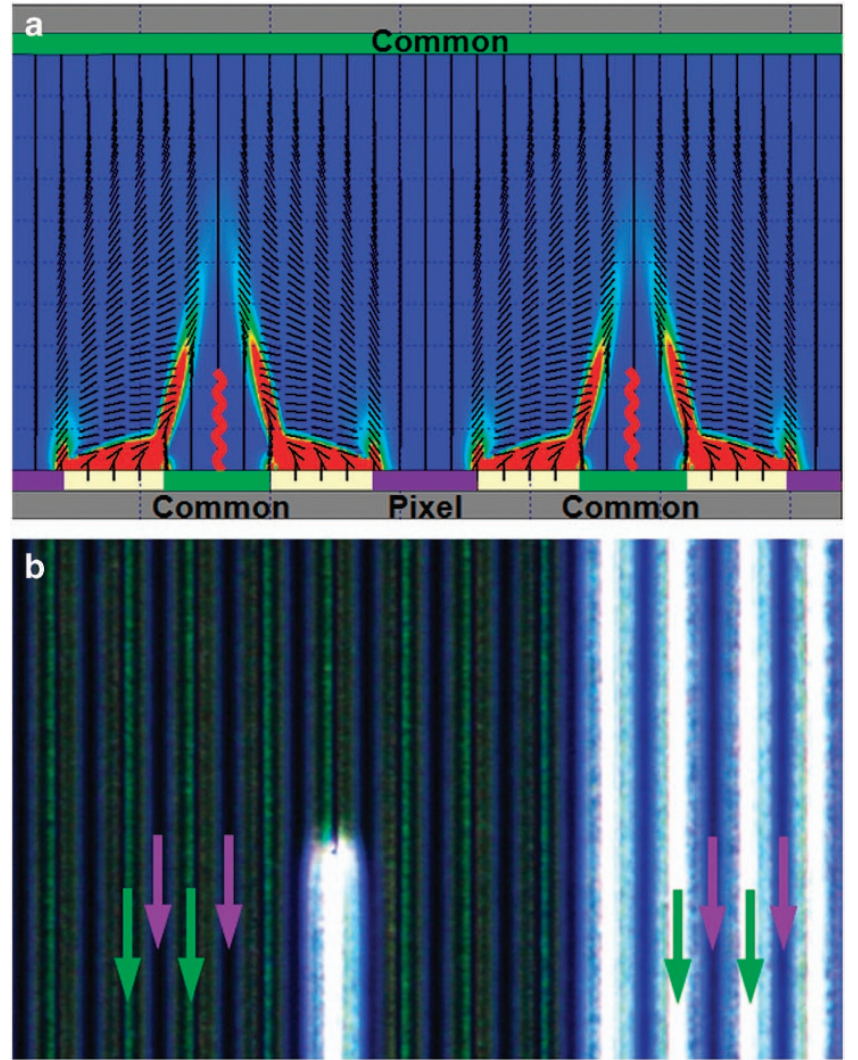

Figure 3 (a) Calculated director profile with associated elastic energy density of the host liquid crystals (LCs) at $16.0 \mathrm{~V}_{\mathrm{pp}}$ and (b) polarized optical microscopy (POM) texture exhibiting the transition of the polarized optical pattern at $16.0 \mathrm{~V}_{\mathrm{pp}}$. In (a), LC molecules are designated by the thin rods and elastic energy is represented by the color scale. Blue and red correspond to the minimum and maximum elastic energy density, respectively. In (b), green and purple arrows indicate the center of the common and pixel (signal) electrodes on the bottom substrate. The relatively darker stripes on the left randomly transform line by line into the brighter ones on the right. The periodicity of the patterns corresponds to $32.0 \mu \mathrm{m}$ that is double that of the electrode pattern.
Over time, large changes in the POM textures were observed. A much brighter line formed on top of the common electrode and propagated along the disclination line, as shown in Figure $3 \mathrm{~b} .{ }^{36,37}$ This phenomenon occurred randomly line by line, and eventually the brighter line replaced all green disclination lines throughout the cell. The formation of a much brighter POM image implies that a higher degree of in-plane orientation and elastic deformation of the LCs occurred through the transition. A similar transition has been reported for display applications with a positive $\Delta \varepsilon$ LC and in-plane electrodes with no electrode on the top substrate. ${ }^{36,37}$ Further details regarding this transformation can be found in the Supplementary Information. Once the transformation was complete over the entire cell, the RMs were polymerized as described in the Materials and Methods.

Similar to the results presented in Figure 1, the periodic optical pattern of the LC host was stabilized by the polymerized RM networks. Both the optical and morphological patterns of the networks were templated by the periodically deformed LC host. Supplementary Figure S7 in the Supplementary Information presents the results obtained for a combined cell. Figure 4 shows structured networks bound to the top substrate (that is, far side of UV range) after disassembling the cell. Although unpatterned ITO glass was used for the top substrate, an RM network with a regular pattern was formed with twofold the periodicity of the ITO pattern of the bottom substrate. The green and purple dots in the figures correspond to the common and signal electrodes on the opposing substrate, respectively. By referring to Figures $4 \mathrm{a}$ and $\mathrm{b}$, it is evident that a residual amount of networked material was formed on the signal electrode (purple dot) and dense homeotropic bundles were located above the center of the common electrode (green dot). Most of the birefringent networks were formed above, near the edges of the common electrode. The inplane component of the optical axes of the local networks lay in the transverse to the electrode (that is, along the direction of the in-plane electric field). The SEM images in Figures $4 \mathrm{c}$ and d reveal the details of the network morphology. The network density exhibits high spatial contrast, forming a morphological pattern. As shown in Figure 4d, adjacent cylindrical channels, formed above, near the edges of the common electrode, were divided by a vertical polymer wall, located above the center of the common electrode. The pattern was uniform throughout the sample. The fibril-like morphology of the local networks precisely replicated the local director orientation (that is, optical birefringence) of the host LCs.

Figure 5 shows that structured networks formed on the bottom substrate with patterned electrodes. The polarized optical image in Figure 5a shows a pattern similar to that observed on the opposite substrate (Figure 4a). The pattern is characterized by dark bands on the electrodes and birefringent networks near the edges of the common electrode. The optical image in Figure $5 \mathrm{~b}$ confirms the existence of a dense polymer bundle at the center of the common electrode (marked by green dots) and a substantial amount of networked material on the signal electrode (marked by purple dots). These observations verify the vertically aligned optical axes of the networks on both electrodes. Figure $5 \mathrm{c}$ and the inset show SEM images of the patterned networks with a layered structure. A large amount of networked material formed as a curved layer above the interelectrode area. The spatial distribution of the networks precisely matched the profile of the highly deformed regions of the host LCs, as shown in red in Figure 3a and Supplementary Figure S3. The optical axes of the local networks were replicated by the local director of the LC host.

To investigate the surface networks shown in Figures $5 \mathrm{a}-\mathrm{c}$ in greater detail, the upper networks were carefully removed using adhesive tape. 

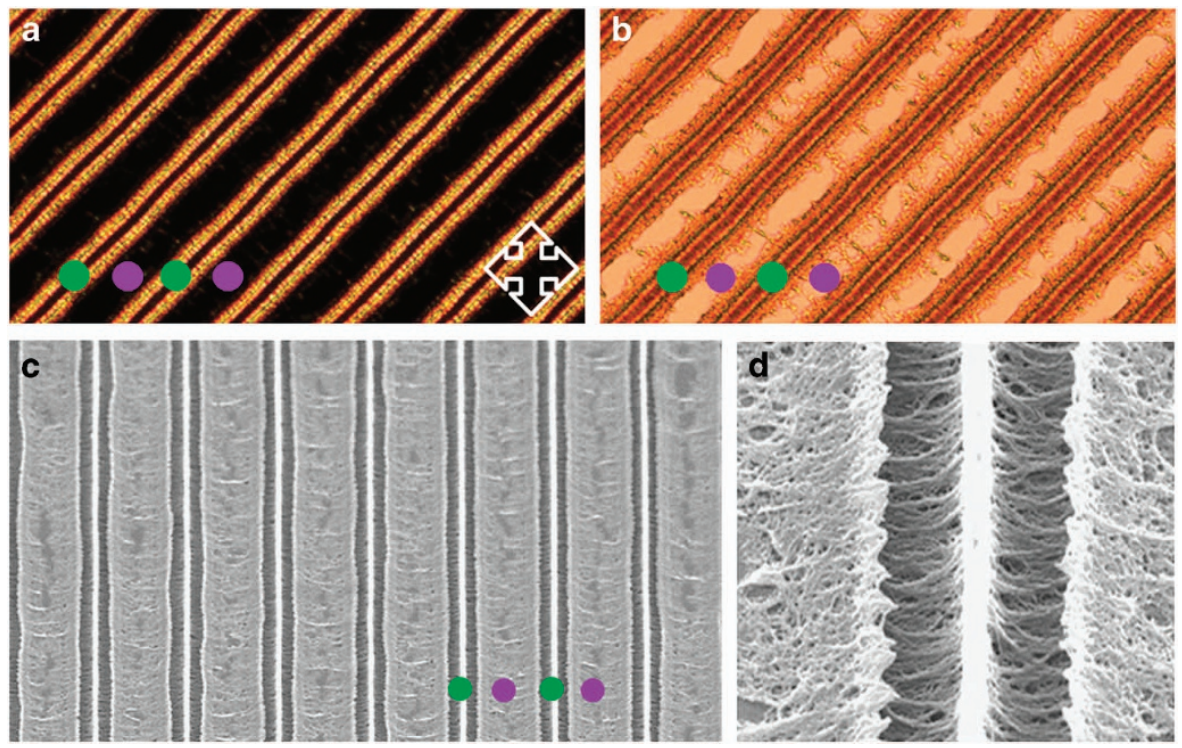

Figure 4 (a) Polarized optical, (b) optical and (c, d) scanning electron micrographs of the reactive mesogen (RM) networks formed on the top substrate with an unpatterned indium tin oxide (ITO) electrode. Green and purple dots designate the corresponding locations of the common and signal electrodes on the bottom substrate. The transmission axis of the crossed polarizers is denoted by the double-ended arrows in (a). In (a-d), the periodicity of the polymer patterns corresponds to $32.0 \mu \mathrm{m}$.
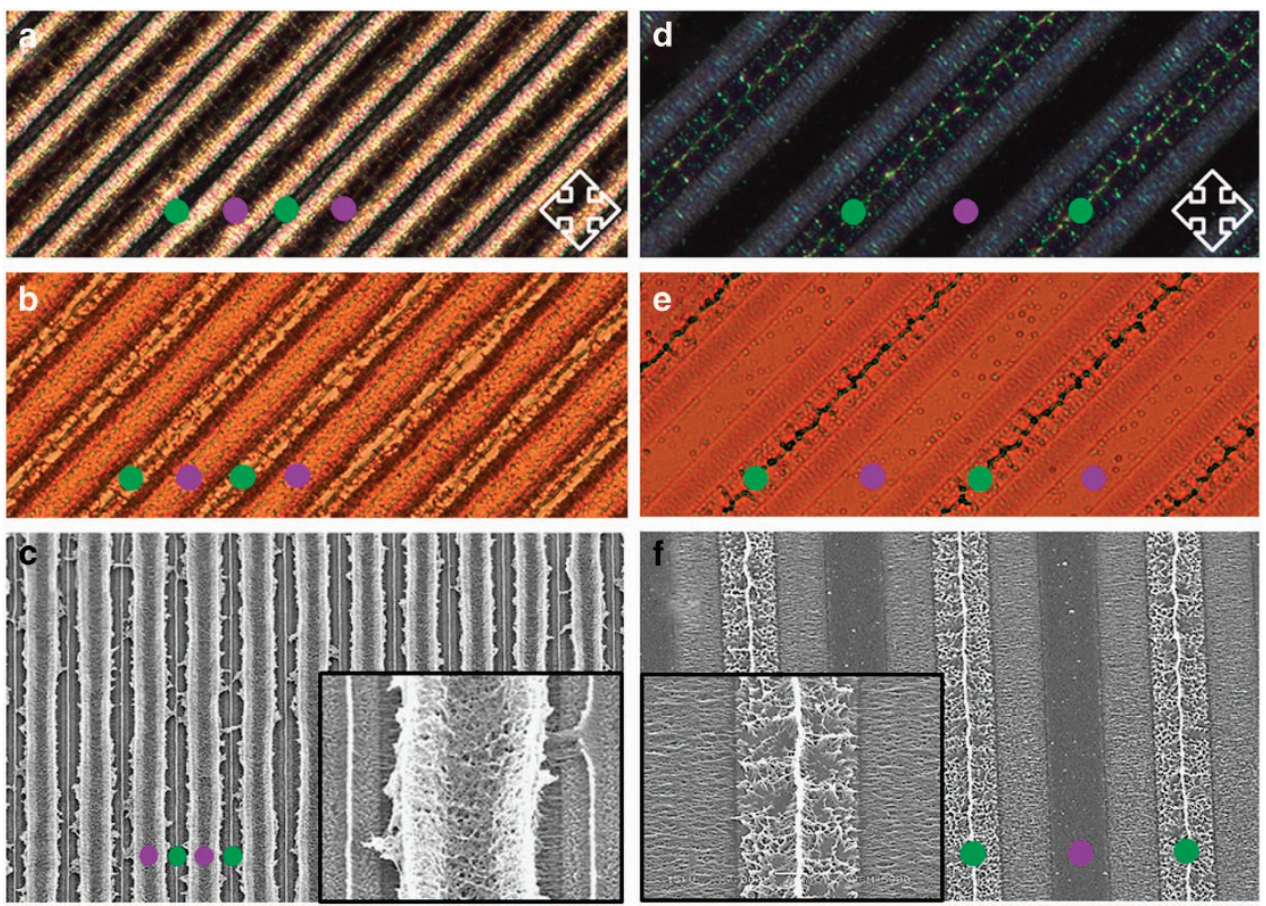

Figure 5 Optical, polarized optical and scanning electron micrographs of the reactive mesogen (RM) networks, formed on the bottom substrate with patterned indium tin oxide (ITO) electrodes. (a-c) Pristine networks observed after the removal of liquid crystal (LCs), and (d-f) templated surface networks adhered to the patterned electrodes. The surface networks were observed after removal of the dense curved networks shown in Figure $6 \mathrm{c}$. The insets in (c, f) present magnified views of the patterns. Green and purple dots represent the locations of the common and signal electrodes. The transmission axis of the polarizers is denoted by the double-ended arrows in (a, d). The periodicity of the polymer patterns corresponds to $32.0 \mu \mathrm{m}$.

POM and SEM images of the surface networks strongly adhered to the bottom substrate as shown in Figures 5d-f. No polymer network was observed on the signal electrode (marked by purple dots). In the interelectrode area, fine fibrils aligned transverse to the electrodes formed a thin surface layer. As confirmed by the polarized optical and optical images in Figures $5 \mathrm{~d}$ and e, the optical axis of the network was parallel to the fibril direction. On the common electrode, however, the polymer fibrils, which formed a dense wall at the center, were aligned predominantly normal to the surface, as shown in the inset of the SEM image (Figure 5f). All observed morphologies and optical birefringences of the networks corroborate the director configuration of host LCs near the surface (see Figure 3a and Supplementary Figure S3). 

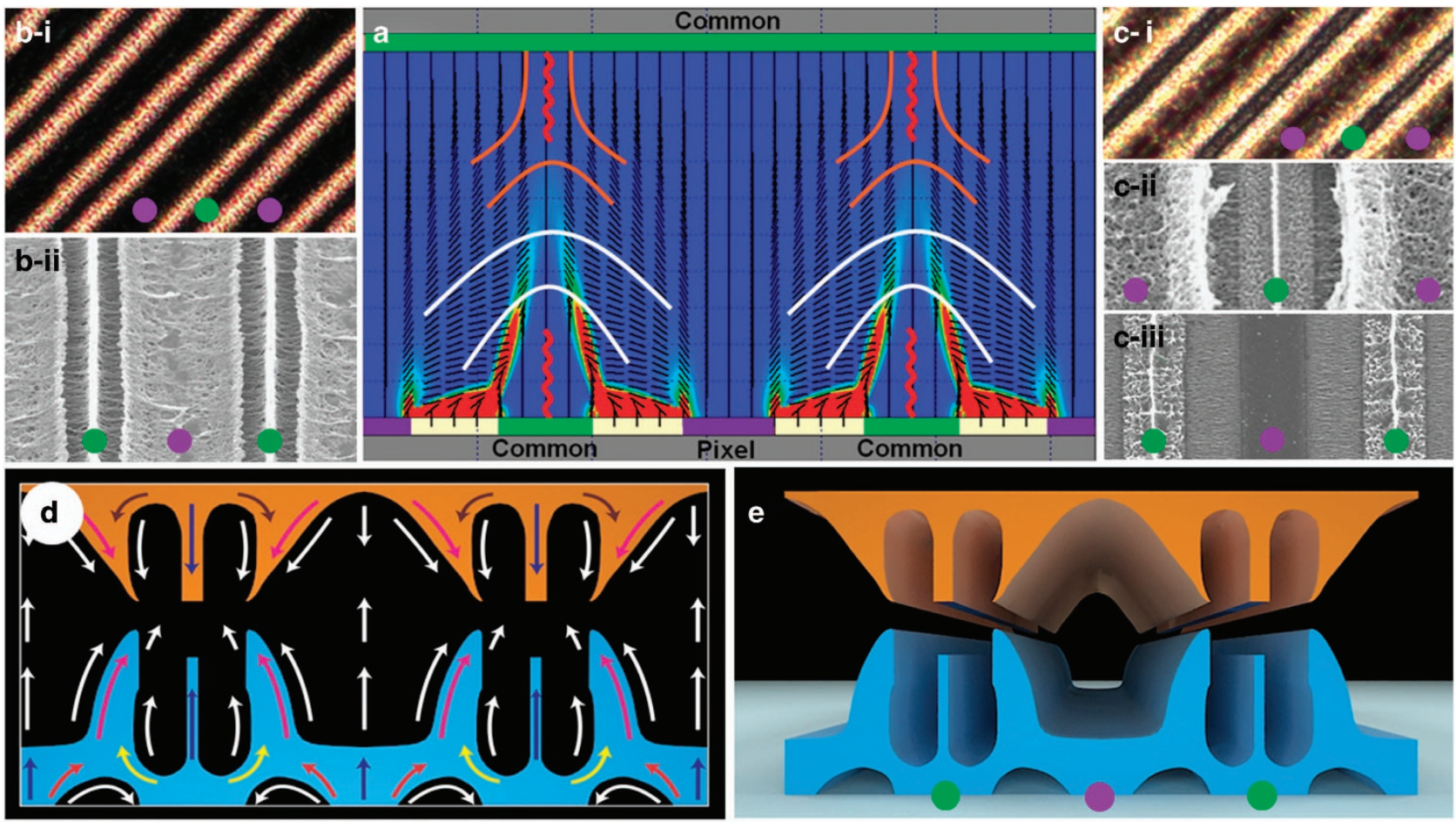

Figure 6 Three-dimensionally (3D)-architectured reactive mesogen (RM) networks formed at $16.0 \mathrm{~V}_{\mathrm{pp}}$. (a) Calculated director configuration with associated elastic deformation of the host liquid crystals (LCs), polarized optical microscopy (POM) and scanning electron microscopy (SEM) images observed on the unpatterned top substrate (bi, bii) and formed on the patterned bottom substrate (ci-ciii), (d) cross-sectional view of the reconstructed RM networks with the local director orientations and (e) 3D view of the reconstructed RM networks. Green and purple dots indicate the locations of the common and pixel (signal) electrodes on the bottom substrate. In (a), white and pink solid lines illustrate the director profiles with the disclinations (shown by the red wavy lines) bound to both the top and bottom substrates. The pattern in (ciii) corresponds to the surface layer of the networks shown in (cii). White and colored arrows in (d) represent the local director orientation of the host LCs and RM networks, respectively. The periodicity of the polymer patterns corresponds to $32.0 \mu \mathrm{m}$.

Figure 6 shows the 3D-architectured polymer networks constructed by photopolymerizing RMs in a periodically deformed nematic liquid crystal as a reaction medium. Figure 6 a illustrates the director profile, the associated elastic energy and the formation of disclinations at $16.0 \mathrm{~V}_{\mathrm{pp}}$. The textural transformation, discussed with respect to Figure $3 \mathrm{~b}$ and the Supplementary Information, cannot be understood based solely on the theoretical calculations. Because of the boundary conditions and topological theory applied to defects, the observed transformed state cannot be obtained by computer simulation. ${ }^{40}$ Based on our observations and previous reports, ${ }^{36,37}$ we infer that the disclination wall, anchored at the center of the bottom common electrode (Supplementary Figure S6), split into two disclination walls, each residing on opposing substrates, as illustrated by the red wavy lines in Figure 6a. Because of strong dielectric coupling, the elastic deformation became stronger and extended toward the top substrate in the common electrode and interelectrode regions. The extended disclination wall was suppressed through structural transformation, as discussed in the Supplementary Information. Eventually, disclination walls could be mitigated by localization and were bound to each substrate through the transition, as depicted in Figure 6a (see also Supplementary Figures S4-S6 in the Supplementary Information).

The POM and SEM results well support our inference. The polarized optical and morphological patterns shown in Figures $6 \mathrm{~b}$ and c correspond to the structured RM networks formed on the top and bottom substrates, respectively. The green and purple dots indicate the locations of the common and signal electrodes on the bottom substrate. Figures $6 \mathrm{a}-\mathrm{c}$ clearly demonstrate that the spatial distribution of the networks was determined by the corresponding elastic deformation and that the orientational order of the networks was templated by the local director of the host LCs. Figure $6 \mathrm{~d}$ shows a cross-sectional view of the reconstructed $3 \mathrm{D}$ networks. The LC host occupies the dark region, and its director orientation is denoted by the white arrows. POM and SEM imaging allowed for the identification of the local molecular order of the RM networks, indicated by the colored arrows. The corresponding 3D view of the reconstructed networks is presented in Figure 6e. The figure shows an elaborately interconnected and unique 3D structure with the embedded pattern of molecular orientational order (that is, optical birefringence).

At a low applied voltage of $4.0 \mathrm{~V}_{\mathrm{pp}}$, structured networks formed near the edges of the electrodes, where most of the elastic deformation occurred. However, polymerization in a more spatially deformed reaction medium at a high voltage $\left(16.0 \mathrm{~V}_{\mathrm{pp}}\right)$ resulted in unique $3 \mathrm{D}$ architectured polymer networks with high spatial contrast and fidelity. The results for both low (Figure 2) and high (Figure 6) applied voltage unambiguously demonstrate that the spatial and orientational templating of polymerized networks can be achieved in an elastically deformed LC host as a polymerization medium. Studies aimed at formulating a more detailed mechanism for the phase separation process and elastic energy-driven templating procedures are currently underway.

\section{CONCLUSIONS}

In conclusion, it was demonstrated that spatially and orientationally patterned polymer architectures can be constructed by photopolymerizing RMs in periodically deformed liquid crystals. Without using lithographic or holographic implements, various $2 \mathrm{D}$ or $3 \mathrm{D}$ polymer patterns can be produced by employing nematic LCs as reaction solvents and spatially nonuniform electric fields with patterned electrodes. Deliberately planned experiments and computer simulations clearly 
support our conclusions on the formation of polymer architectures with embedded orientational order at a molecular level. The gradient in elastic energy, induced by a spatially nonuniform electric field and corresponding director configuration, is the driving force for the morphologically patterned phase separation of the RM networks. Our results provide a unique and versatile method for constructing 3D architectures of polymer networks with optical birefringence. Functional soft materials, combining both mesogenic properties and reactivity, can be polymerized into predetermined organized patterns with an incorporated molecular order. This can be achieved by employing LC cells with various types of patterned electrodes (that is, a reaction vessel) and liquid crystals as the reaction medium. Therefore, the proposed method is useful for processing functional soft materials with morphological and orientational anisotropy to enhance their functional performance.

\section{CONFLICT OF INTEREST}

The authors declare no conflict of interest.

\section{ACKNOWLEDGEMENTS}

This research was supported by the 'BK21 Plus Project' through the National Research Foundation of Korea funded by the Ministry of Education. We thank the SANAI System for their support and discussion of the computation of the director configuration and elastic energy profile.

\section{PUBLISHER'S NOTE}

Springer Nature remains neutral with regard to jurisdictional claims in published maps and institutional affiliations.

1 Nalwa, H. S. in Handbook of Advanced Electronic and Photonic Materials and Devices Vol. 8 (ed Nalwa H. S.) 185-208 (Academic Press: New York, London, Sydney, Tokyo, 2001)

2 Xia, Y. \& Whitesides, G. M. Soft lithography. Annu. Rev. Mater. Sci. 28, 153-184 (1998).

3 Alfred, M. P., Contoret, A. E. A., Farrar, S. R., Kelly, S. M., Mathieson, D., O'Neil, M., Tsoi, W. C. \& Vlachos, P. A full color-electroluminescent device and patterned photoalignment using light emitting-liquid crystals. Adv. Mater. 17, 1368-1372 (2005).

4 Nie, Z. \& Kumacheva, E. Patterning surfaces with functional polymers. Nat. Mater. 7, 277-290 (2008)

5 Gather, M. C., Koehnen, A., Falcou, A., Beker, H. \& Meerholz, K. Solution-processed full-color polymer organic light-emitting diode displays fabricated by direct photolithography. Adv. Funct. Mater. 17, 191-200 (2007).

6 Muller, C. D., Falcou, A., Reckefuss, N., Rojahn, M., Wiederhirn, V., Rudati, P., Frohne, H., Nuyken, O., Becker, H. \& Meerholz, K. Multi-colour organic light-emitting displays by solution processing. Nature 421, 829-833 (2003).

7 Yoon, D. K., Choi, M. C., Kim, Y. H., Kim, M. W., Lavrentovich, O. D. \& Jung, H.-T. Internal structure visualization and lithographic use of periodic toroidal holes in liquid crystals. Nat. Mater. 6, 866-870 (2007).

8 Honglawan, A., Beller, D. A., Cavallaro, M., Kamien, R. D., Stebe, K. J. \& Yang, S. Pillar-assisted epitaxial assembly of toric focal conic domains of smectic-A liquid crystals. Adv. Mater. 23, 5519-5523 (2011).

9 Li, Q. in Anisotropic Nanomaterials (ed. Li Q.) Ch. 6 \& 7 (Springer: Switzerland, 2015).

10 Li Q. in Nanoscience with Liquid Crystals (ed. Li Q.) Ch. 5 \& 6 (Springer: Switzerland, 2014).

11 Drzaic P. S.in Liquid Crystal Dispersions (ed. Drzaic P. S.) Ch. 4 \& 5 (World Scientific: Singapore, 1995).

12 Crawford, G. P., Zumer, S. in Liquid Crystals in Complex Geometry (eds Crawford G. P. \& Zumer S.) Ch. 3, 4, \& 5 (Taylor \& Francis: Bristol, PA, USA, 1996).

13 Broer, D. J., Crawford, G. P., Zumer, S. in Cross-Linked Liquid Crystalline Systems (eds Broer D. J., Crawford G. P. \& Zumer S.) Ch. 2 \& 6 (CRC Press (Taylor \& Francis Group): Boca Rato, FL, USA, 2011).

14 Kikuchi, H., Yokota, M., Hisakado, Y., Yang, H. \& Kajiyama, T. Polymer-stabilized liquid crystal blue phases. Nat. Mater. 1, 64-68 (2002).

15 Araya, K., Mukoh, A., Narahara, T. \& Shirakawa, H. Polymerization of acetylene in liquid crystal solvent. Chem. Lett. 13, 1141-1142 (1984).

16 Noh, J. H., Henx, B. \& Lagerwall, J. P. F. Taming liquid crystal self-assembly: the multifaceted response of nematic and smectic shells to polymerization. Adv. Mater. 28, 10170-10174 (2016).
17 Hikmet, R. A. M. \& Kemperman, H. Electrically switchable mirrors and optical components made from liquid-crystal gels. Nature 392, 476-479 (1998).

18 Khandelwal, H., Loonen, R., Hensen, J., Debije, M. \& Schenning, A. Electrically switchable polymer stabilised broadband infrared reflectors and their potential as smart windows for energy saving in buildings. Sci. Rep. 5, 11773-11782 (2015).

19 Hikmet, R. A. M. Anisotropic gels and plasticized networks formed by liquid crystal molecules. Liq. Cryst. 9, 405-416 (1991).

20 Hulvat, J. F. \& Stupp, S. I. Liquid-crystal templating of conducting polymers. Angew. Chem. Int. Ed. 42, 778-781 (2003).

21 Hikmet, R. A. M. \& Polesso, R. Patterned multicolor switchable cholestric liquid crystal gels. Adv. Mater. 14, 502-504 (2002).

22 Witte, P., Brehmer, M. \& Lub, J. LCD components obtained by patterning of chiral nematic polymer layers. J. Mater. Chem. 9, 2087-2094 (1999).

23 Zhang, J., Carlen, C. R., Palmer, S. \& Sponsler, M. B. Dynamic holographic grating recorded by photopolymerization of liquid crystalline monomers. J. Am. Chem. Soc. 116, 7055-7063 (1994).

24 Tondiglia, V. P., Natarajan, L. V., Sutherland, R. L., Tomlin, D. \& Bunning, T. J. Holographic formation of electro-optical polymer \pm liquid crystal photonic crystals. Adv. Mater. 14, 187-191 (2002).

25 Penterman, R., Klink, S. I., Koning, H., Nisato, G. \& Broer, D. J. Single-substrate liquid-crystal displays by photo-enforced stratification. Nature 417, 55-58 (2002).

26 Broer, D. J., Lub, J. \& Mol, G. N. Wide-band reflective polarizers from cholestric polymer networks with a pitch gradient. Nature 378, 467-469 (1995).

27 Broer, D. J., Mol, G. N., Haaren, J. A.M.M. \& Lub, J. Photo-induced diffusion in polymerizing chiral-nematic media. Adv. Mater. 11, 573-578 (1999).

28 Kang, S.-W., Sprunt, S. \& Chien, L. C. Polymer-stabilized cholesteric diffraction gratings: effects of UV wavelength on polymer morphology and electrooptic properties. Chem. Mater. 18, 4436-4441 (2006).

29 Kang, S.-W., Sprunt, S. \& Chien, L. C. Photoinduced localization of orientationally ordered polymer networks at the surface of a liquid crystal host. Macromolecules 35, 9372-9376 (2002).

30 Kang, S.-W., Sprunt, S. \& Chien, L. C. Ordered polymer microstructures obtained usingpattern forming states of a cholesteric liquid crystal as templates. Adv. Mater. 13, 1179-1182 (2001)

31 Kang, S.-W., Jin, S. H., Chien, L. C. \& Sprunt, S. Spatial and orientational templating of semiconducting polymers in a cholestric liquid crystal. Adv. Func. Mater. 14, 329-334 (2004).

32 Goto, H. Doping-dedoping-driven optic effect of conjugated polymers prepared in cholesteric-liquid-crystal electrolytes. Phy. Rev. Lett. 98, 253901 (2007).

33 Goh, M., Matsushita, T., Satake, H., Kyotani, M. \& Akagi, K. Macroscopically aligned helical polyacetylene synthesized in magnetically oriented chiral nematic liquid crystal field. Macromolecules 43, 5943-5948 (2010).

34 Jiao, M., Ge, Z., Wu, S. T. \& Choi, W. K. Submillisecond response nematic liquid crystal modulators using dual fringe field switching in a vertically aligned cell. J. Appl. Phys. 92, 111101-111103 (2008).

35 Kim, M. S., Seen, S. M., Jeong, Y. H., Kim, H. Y., Kim, S. Y., Lim, Y. J. \& Lee, S. H. Dynamic stability of disclination lines in fringe-field switching mode with a wedgeshaped common electrode. J. J. Appl. Phys. 45, 883-886 (2006).

36 Lim, Y. J., Choi, Y. E., Lee, J. H., Lee, G.-D., Komitov, L. \& Lee, S. H. Effects of threedimensional polymer networks in vertical alignment liquid crystal display controlled by in-plane field. Opt. Express 22, 10634-10641 (2014).

37 Chen, T. J., Lin, G. J., Chen, B. Y., Lin, B. R., Wu, J. J. \& Yang, Y. J. Optimized electrooptical properties of polymer-stabilized vertical-aligned liquid crystal displays driven by an in-plane field. Displays 37, 94-99 (2015).

38 Vitale, A., Hennessy, M. G., Matar, O. K. \& Cabral, J. T. Interfacial profile and propagation of frontal photopolymerization waves. Macromolecules 48, 198-205 (2015).

39 Pojman, J. A. in Frontal Polymerization Vol. 4 (eds Matyjaszewski K. \& Moller M.) Ch. 4.38, 957-977 (Elsevier B. V., Amsterdam, Netherland, 2012).

40 Kleman, M. \& Lavrentovich, O. D. in Soft Matter Physics (eds Kleman M. \& Lavrentovich O. D.) 434-467 (Ch. 12) (Springer: NY, USA, 2003).

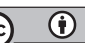

This work is licensed under a Creative Commons Attribution 4.0 International License. The images or other third party material in this article are included in the article's Creative Commons license, unless indicated otherwise in the credit line; if the material is not included under the Creative Commons license, users will need to obtain permission from the license holder to reproduce the material. To view a copy of this license, visit http:// creativecommons.org/licenses/by/4.0/

(C) The Author(s) 2017

Supplementary Information accompanies the paper on the NPG Asia Materials website (http://www.nature.com/am) 